\title{
Intension, extension, and the model of belief and knowledge in economics
}

\author{
IVAN MOSCATI \\ University of Insubria \\ Bocconi University
}

\begin{abstract}
This paper investigates a limitation of the model of belief and knowledge prevailing in mainstream economics, namely the state-space model. Because of its set-theoretic nature, this model has difficulties in capturing the difference between expressions that designate the same object but have different meanings, i.e., expressions with the same extension but different intensions. This limitation generates puzzling results concerning what individuals believe or know about the world as well as what individuals believe or know about what other individuals believe or know about the world. The paper connects these puzzling results to two issues that are relevant for economic theory beyond the state-space model, namely, framing effects and the distinction between the model-maker and agents that appear in the model. Finally, the paper discusses three possible solutions to the limitations of the state-space model, and concludes that the two alternatives that appear practicable also have significant drawbacks.
\end{abstract}

Keywords: intension, extension, belief, knowledge, interactive belief, state-space model

JEL Classification: B41, C70, D80

Much of current mathematical economic analysis is based, in one way or another, on the theory of sets initiated by Georg Cantor (1883) and Richard Dedekind (1888) in the late nineteenth century, axiomatically developed by Ernst Zermelo (1908), Abraham Fraenkel (1923), John von Neumann (1928), and others in the early twentieth century, and usually

AuThOR's Note: I am grateful to Robert Aumann, Pierpaolo Battigalli, Giacomo Bonanno, Richard Bradley, Paolo Colla, Marco Dardi, Alfredo Di Tillio, Vittorioemanuele Ferrante, Francesco Guala, Conrad Heilmann, and Philippe Mongin for helpful discussions and suggestions on previous drafts. The paper has also benefited from the many insightful comments made by three anonymous referees and co-editor François Claveau. I also thank the participants at seminars at Bocconi University and LSE for helpful comments. Finally, I am grateful to Bocconi University for financial support, and to the Centre for Philosophy of Natural and Social Science at LSE for its hospitality during part of the work on the paper. Any errors are mine. 
known as Zermelo-Fraenkel set theory. In the set-theoretic mathematical approach, the objects of economic interest are conceptualized as elements of some set, e.g., the set of commodity bundles or the set of actions available to a certain player. The features of these sets, e.g., their being closed or convex, are typically attributed with some important economic meaning. Preferences, technologies, strategies, and other economically relevant notions are modeled as functions that associate the elements of one set with the elements of another set. ${ }^{1}$

The model of belief and knowledge prevailing in contemporary mainstream economics and used especially in information economics and game theory is also set-theoretic in nature. This model was introduced by Robert Aumann (1976); it formalizes beliefs and knowledge as two set-theoretic operators $B$ and $K$ that map subsets of a universal space $\Omega$ into other subsets of the same space. Aumann's model and its generalizations have been variously labeled as "the eventbased approach to belief and knowledge", "possibility correspondence model", "possible world semantics", "Aumann structures", and "the state-space model of belief and knowledge". This last name will be adopted here. In the state-space model, the difference between belief and knowledge is that, while belief can be false, knowledge is assumed to be truthful. In other words, knowledge is a special case of belief, namely true belief. ${ }^{2}$

As any other economic model, the state-space model displays a number of limitations. Some of them have been discussed already in the philosophical, economic, and artificial intelligence literature under the banner of the logical omniscience problem. In particular, it has been pointed out that the model implies the monotonicity of beliefs and knowledge, i.e., that an individual believes or knows the implications of what she believes or knows. For instance, if an individual knows the axioms of a mathematical system, monotonicity says that she also knows all the theorems that are valid in the system (Fagin, et al. 1995, ch. 9). Another implausible feature of the state-space model is that it rules out the realistic possibility that an individual may be completely

\footnotetext{
${ }^{1}$ On the history of set theory and how it spread into economics, see Ferreirós 2007; Giocoli 2001; Weintraub 2002.

${ }^{2}$ For philosophers, the truthfulness of belief is a necessary but not sufficient condition for knowledge, since the latter requires that the true belief is justified. A number of examples originally put forward by Gettier (1963) have suggested that even justified true belief does not warrant proper knowledge. For an introductory discussion of the definition of knowledge as justified true belief and its refinements, see Steup 2012.
} 
unaware of an event rather than believing or not believing it (Dekel, et al. 1998).

In this paper, I focus on a less explored limitation of the state-space model, which is strictly related to its set-theoretic nature and, more specifically, to the first axiom of the Zermelo-Fraenkel set theory. This first axiom is usually labeled as the axiom of extensionality and it states that two sets are equal if and only if they collect the same elements, i.e., that the extension of a set as defined by the elements belonging to it fully characterizes the set. A consequence of this axiom is the so-called principle of substitutivity. According to this principle, if two sets collect the same elements, whatever holds true for one set also holds true for the other, and thus one set can be substituted for the other salva veritate (preserving truth).

The axiom of extensionality and the principle of substitutivity are less obvious than appears at first sight. At least since the late nineteenth century, philosophers such as Gottlob Frege (1948 [1892]) have pointed out that in a number of contexts the substitutivity principle may fail. Among these contexts are those involving beliefs and knowledge. For instance, although the expressions "Morning Star" and "Evening Star" identify the same planet, i.e., Venus, an individual may (truthfully) believe that the planet that appears in the eastern part of the sky in the morning is Venus, while she (erroneously) believes that the planet that appears in the western part of the sky in the evening is not Venus.

These kinds of situations can be conceptualized through the notions of intension and extension (Carnap 1947). The intension of a linguistic expression is what the expression means, i.e., the notion or idea it conveys. The extension of an expression is the set of things it designates or applies to. So, for instance, the intension of the term "computer" is the idea of an electronic machine that can store, retrieve, and process data, while its extension is the set of existing computers. In the astronomical example above, we have two expressions that have different intensions ("Morning Star" conveys a different meaning from "Evening Star") but the same extension (the planet Venus). Using the intension-extension terminology, Frege and others drew attention to the concern that the substitutivity principle may be invalid for beliefs and knowledge involving expressions with the same extension but different intensions, and therefore its unchecked application can lead to mistaken conclusions. 
The limitation of the state-space model I focus on in this paper is that the operators $B$ and $K$ automatically implement the substitutivity principle also for expressions with the same extension but different intensions, that is, also if substitutivity is not warranted. In the first place, this feature of $B$ and $K$ generates puzzling results that concern what an individual believes or knows about the world. Furthermore, that feature of $B$ and $K$ generates puzzling results pertaining to interactive beliefs and knowledge, i.e., the beliefs and knowledge that an individual has about what other individuals believe or know about the world. In particular, the model may imply that an individual knows that another individual believes a certain event, even independently of any specific assumption about what the former individual knows about the way the information is imparted to the latter. I connect these puzzling results to two issues that are relevant for economic theory, namely those concerning framing effects and the distinction between the viewpoint of the model-maker and the viewpoint of the agents in the model.

In the final section, I discuss three possible ways of modifying the operators $B$ and $K$ and/or the state-space model, in order to make them capable of capturing the difference between intensions with the same extension. While a first approach based on a re-definition of $B$ and $K$ appears barren, the other two lines of attack-re-defining the universal space $\Omega$ or adopting the so-called syntactic approach-are more promising but they also present significant drawbacks.

The difficulties that standard set-theoretic economic models have in capturing the difference between different intensions with the same extension have rarely been discussed explicitly in the economics literature. I am aware of four exceptions. Kenneth Arrow $(1982,6)$ mentioned that, contrary to the implicit assumption of standard economic theory, preferences for commodities may violate the axiom of extensionality. Michael Bacharach (1986, 182-183) added that, contrary to standard probability and decision theory, beliefs may also violate extensionality. Arnis Vilks (1995, 195-199) pointed out that standard mathematical economics is rooted in the extensional setting of the Zermelo-Fraenkel set theory, and therefore is ill-suited for modeling preferences and beliefs, for which extensionality may not hold. Sacha Bourgeois-Gironde and Raphaël Giraud (2009) connected framing effects with violations of extensionality and proposed by-passing 
extensionality through a modified version of the so-called Bolker-Jeffrey decision model.

The present paper expands on these previous contributions in a numbers of ways: it analyzes in detail how the extensional nature of $B$ and $K$ generates puzzling consequences in the state-space model; it calls attention to the puzzling implications that the state-space formalism has in relation to interactive beliefs and knowledge; it brings into play the notion of intension and shows how the intension-extension dichotomy helps us to better understand the limitations of the statespace model; finally, it discusses some possible solutions to the limitations of $B, K$, and the model.

It is important to stress that the goals of the paper are "informative" and "diagnostic", rather than "therapeutic". The paper has an informative aim in the sense that it calls the attention of economists to problems related to the relationship between intension and extension that, although typically ignored in the economic literature, are relevant for economic analysis. The paper is diagnostic in that it explains why the state-space model has difficulties in dealing with the intensionextension difference. The paper also ventures into the therapeutic realm by discussing some possible solutions to these difficulties. However, it does not provide a systematic solution to the problems that it calls attention to.

Section 1 provides some further insights from the philosophical literature on the relationship between intension and extension, and shows how these insights can be used to conceptualize framing effects and the distinction between model-maker's and agents' viewpoints in the model. Section 2 reviews the state-space model of belief and knowledge. Section 3 illustrates how the set-theoretic nature of the operators $B$ and $K$ generates puzzling results concerning what an individual believes or knows about the world. Section 4 investigates the puzzling implications of the state-space model relative to interactive beliefs and knowledge. Section 5 discusses possible solutions to the limitations of $B, K$, and the state-space model. Section 6 sums up the paper.

\section{ON INTENSION AND EXTENSION}

As mentioned in the Introduction, philosophers have explored issues related to the relationship between intension and extension at least 
since the late nineteenth century. ${ }^{3}$ These explorations are related to philosophical efforts to clarify the proper content and the cognitive value of linguistic expressions and thoughts. One may even say that large parts of twentieth-century philosophy of language and of mind are somehow related to issues concerning the relationships between intension and extension. I will not attempt to provide an overview of this immense literature. ${ }^{4}$ Instead, I will mention some insights from this philosophical literature that may help the reader with an economic background to see the larger set of problems that the issues addressed in this paper relate to, and help the reader with a philosophical background to connect the paper with questions with which she is already familiar.

In the first place, situations involving beliefs and knowledge belong to a larger family of cases in which the relationship between intension and extension can be tricky. In the current philosophy of language and of mind these cases are usually labeled as "referentially opaque contexts". Besides those involving beliefs and knowledge, other referentially opaque contexts are those involving propositional attitude verbs like "prefers", "desires", "hopes", "wants", or "says"; in these contexts the substitutivity principle may also fail: Ann may desire to see the Morning Star while she does not desire to see the Evening Star. Other referentially opaque contexts where the substitutivity principle could become problematic are those in which modal verbs such as "it is necessary that" or "it is possible that" are used. For instance, although it is true that "the number of planets in the solar system is 8 ", and that " 8 is necessarily greater than 7 ", it is false that "the number of planets is necessarily greater than 7 ".

Furthermore, the reference of an expression can be opaque not only because there are extensions with multiple intensions as in the cases mentioned above, but also because there are intensions without any actual extension, as in the case of expressions indicating fictional entities such as "unicorn" or "the round square". And there are also intensions whose references are context-dependent and thus opaque, as in the case of indexical expressions such as "I", "that", or "here".

A further point discussed in the philosophical literature is the failure of the substitutivity principle even for so-called hyper-intensional

\footnotetext{
${ }^{3}$ The expressions "intension" and "extension" derive from Carnap (1947). Frege (1948 [1892]) talked, in an analogous manner, of "sense" and "reference".

${ }^{4}$ For an introduction, see Bealer 1998; McKay and Nelson 2010.
} 
propositions-that is, for propositions expressing mathematical truths and other supposedly necessary truths. Consider these additional expressions having different intensions but equal extension: "Tegucigalpa" and "the capital of Honduras", "equilateral triangle" and "equiangular triangle", and a hyper-intensional proposition such as " 51 " and " $17 \times 3$ ". Bob may know that the capital of Honduras is not in Nicaragua while believing that Tegucigalpa is in Nicaragua. Carl could know that the triangle in front of him is equilateral without realizing that it is also equiangular. One might think that the failure of the substitutivity principle in these situations depends on the fact that the extensional equality among those propositions is only accidental, that is, non-necessary. Tegucigalpa and the capital of Honduras have the same extension in the actual geopolitical world, but may have different extensions in another possible world. Equilateral and equiangular triangles coincide in Euclidean geometry, but may differ in some non-Euclidean system. However, the principle of substitutivity can fail even in hyper-intensional contexts where extensional equality holds in every imaginable universe. Thus, although it is always the case that $17 \times 3=51$, David may know that $17 \times 3$ is not prime, but not know that 51 is not prime.

In the last sixty-five years, philosophers and logicians such as Rudolf Carnap (1947), Alonzo Church (1951; 1973; 1974), Richard Montague (1960; 1970), Daniel Gallin (1975), Edward Zalta (1988), Melvin Fitting (2004) and others have developed logical systems called intensional logics that are aimed at capturing explicitly the difference between intension and extension. However, a number of problems still remain unresolved and none of these intensional logics has gained general acceptance (Garson 1998; Fitting 2011).

Although the notions of intension and extension have been used only very rarely in the economics literature, they can be fruitfully employed to conceptualize two issues that are relevant for economic theory, namely those concerning framing effects and the relationship between the viewpoint of the model-maker and the viewpoint of the agents in the model.

Framing effects occur when different descriptions of the same object lead to different beliefs, preferences, or decisions concerning that object. For instance, in a series of well-known experiments performed by Amos Tversky and Daniel Kahneman (1981), a group of individuals was asked to state their preferences between different lotteries. 
A number of these lotteries were identical in terms of final outcomes and probabilities, but some of them were framed as one-stage games and others as two stage-games. ${ }^{5}$ Tversky and Kahneman recorded that a majority of subjects changed their preferences between identical lotteries according to the way these lotteries were framed.

This effect can be aptly expressed in terms of intension and extension: the lotteries Tversky and Kahneman submitted to their experimental subjects were extensionally equivalent with respect to their outcomes and probabilities, but intensionally different because one-stage games and two-stage games frame uncertainty differently. More generally, framing effects may be conceived of as the effects, on beliefs, preferences or decisions, of intensionally different descriptions of an extensionally single object.

Orthodox economists tend to discard framing effects as manifestations of the irrationality of individuals who simply fail to recognize that identical things are indeed identical. ${ }^{6}$ In opposition to this view, Tversky and Kahneman and other behavioral economists have argued that framing effects significantly influence economic behavior and therefore cannot be discarded without weakening the descriptive significance of economic theory; moreover, some framing effects seem to have a rational justification. ${ }^{7}$

If we look at framing effects using the notions of intension and extension, they no longer appear to be manifestations of irrationality. Rather, they seem to be just other instances of the failure of the substitutability principle in referentially opaque contexts. Therefore, when looked at from the intension-extension viewpoint, the relevant problem shifts from the issue concerning the individuals' rationality, to the question of whether standard, set-theoretic economic models are able to capture the intensional difference between extensionally equal objects. In Section 3, we will investigate in more detail why the

\footnotetext{
${ }^{5}$ For instance, Lottery $\mathrm{F}$ was described as a one-stage game offering a 20\% chance to win $\$ 45$, and an $80 \%$ chance to win nothing. Lottery D was described as a two-stage game. In the first stage, Lottery D offered a $75 \%$ chance to end the game without winning anything, and a $25 \%$ chance to move into the second stage. In the second stage, Lottery D offered an $80 \%$ chance to win $\$ 45$, and a $20 \%$ chance to win nothing (Tversky and Kahneman 1981, 455). Simple math shows that the final combinations of outcome and probabilities offered by Lotteries D and F are identical.

${ }^{6}$ For instance, preference change between probabilistically equivalent lotteries is usually associated with inconsistency in dynamic choice. For a discussion, see Wakker 1999.

7 For discussion on this topic, see Kahneman and Tversky 1984; Tversky and Kahneman 1986; Bourgeois-Gironde and Giraud 2009.
} 
set-theoretic operators $B$ and $K$ tend to pass over framing effects in the state-space model.

The notions of intension and extension may also be useful in keeping distinct the information and reasoning abilities possessed by the model-maker from the information and reasoning abilities possessed by the agents in the model. In effect, the model-maker often tends implicitly to endow the agents in the model with the comprehensive information and the sophisticated reasoning abilities she possesses. As a consequence, the agents may display beliefs and behavior that appear puzzling, or the results of the model may in fact depend on a number of hidden assumptions concerning the agents' information and reasoning abilities. ${ }^{8}$

The puzzling results concerning interactive beliefs and knowledge in the state-space model-discussed in Section 4 of this paper-can be seen as depending on the hidden assumptions about the agents' information and reasoning abilities harbored by the state-space model. The point I would like to make here is that one way in which the confusion between the model-maker's and the agent's viewpoints may enter into economic models is through the identification of objects that are extensionally equal for the model-maker but intensionally distinct for the agents. Therefore, the notions of intension and extension may help the model-maker to avoid treating in an identical way objects that are equivalent for her but different for the economic agent, and thus enhance transparent and rigorous economic modeling.

After these extensive introductory considerations, we can finally move to the state-space model and discuss how belief and knowledge are modeled in it.

\section{BELIEF AND KNOWLEDGE IN STATE-SPACE MODEL}

In this section, I first outline the formalism of the state-space model and then explain how its various elements can be interpreted (more detailed presentations of the state-space model can be found in Osborne and Rubinstein 1994; Dekel and Gul 1997; Battigalli and Bonanno 1999; Samuelson 2004).

Consider a set $\Omega$ whose generic element is $\omega$, and a correspondence $P: \Omega \rightarrow 2^{\Omega} \backslash\{\varnothing\}$ that associates each element $\omega \in \Omega$ with a set $P(\omega)$ of

\footnotetext{
${ }^{8}$ For a discussion of these issues in game and decision theory, see Brandenburger 1992; Battigalli and Bonanno 1999; Dardi 2004.
} 
elements of $\Omega$ ( $2^{\Omega}$ is the set of all subsets of $\Omega$ ). Based on $P$, define an operator $B: 2^{\Omega} \rightarrow 2^{\Omega}$ as follows: for every $E \subseteq \Omega, B(E)=\{\omega \in \Omega: P(\omega) \subseteq E\}$.

Set $\Omega$ is the set of the possible states of the world. A state $\omega \in \Omega$ specifies all aspects of the world that are relevant to the situation, such as "it rains" or "individual $i$ has transitive preferences". Relevant aspects of the world may include individuals' beliefs, such as in the case of "individual $i$ believes that it rains" or "individual $j$ believes that individual $i$ believes that it rains". Only one state of the world is the true one, but the individual may be uncertain which one that is. This uncertainty is modeled by a correspondence $P$, which associates each state $\omega$ with the set of states that the individual regards as possible at $\omega$. This is why $P$ is called a possibility correspondence.

The possibility correspondence of an individual expresses formally the way information is imparted to her. In the economics of information, this information may be seen as coming from some signal: when the true state of the world is $\omega$, the individual receives a signal suggesting to her that the true state is in the subset $P(\omega)$ of $\Omega$. In game theory, $\omega$ and $P(\omega)$ may be seen, respectively, as a node in a game of incomplete information and as the information set associated with that node. At any rate, notice that possibility correspondences are just a tool that the external, omniscient model-maker employs to encode and represent individuals' beliefs, not something that these individuals themselves need be aware of.

A subset $E \subseteq \Omega$ is called an event, and can be thought of as the collection of all states that share a certain feature. For instance, the event "it rains" collects all states $\omega \in \Omega$ characterized by rain. Note that, if $P(\omega) \subseteq E$ in all states the individual regards as possible in $\omega$, the event $E$ occurs. The operator $B$ is interpreted as a belief operator: if $\omega \in B(E)$, i.e., if $P(\omega) \subseteq E$, then at $\omega$ the individual believes that the event $E$ occurs, and this is because in every state the individual regards as possible in $\omega$-that is, in $P(\omega)$-the event $E$ occurs. Observe that $B(E)$ is itself an event, the event "the agent believes $E$ ". As such, $B(E)$ may become the object of further belief for another agent. We will return to this in Section 4.

As a simple illustration of the model, suppose that an individual-let us call her Ann-is interested in a variable $v$ that can take values from 1 to 4, like a four sided die, and that each state of the world is completely characterized by the value taken in it by $v$. There are thus four possible states of the world: $\Omega=\left\{\omega_{1}, \omega_{2}, \omega_{3}, \omega_{4}\right\}$. Imagine that the 
possibility correspondence of Ann in $\omega_{1}$ is as follows: $P_{A}\left(\omega_{1}\right)=\left\{\omega_{1}, \omega_{2}\right\}$. This means that if $v=1$, Ann is uncertain whether $v=1$ or $v=2$, and considers both states of the world possible.

Consider now the event $R$ " $v$ is equal to 1 ", which occurs only at state $\omega_{1}$, i.e., $R=\left\{\omega_{1}\right\}$, and the event $S$ " $v$ is not greater than 2 ", which occurs at states $\omega_{1}$ and $\omega_{2}$, i.e., $S=\left\{\omega_{1}, \omega_{2}\right\}$. In $\omega_{1}$, does Ann believe $R$ ? No, she does not, because in $\omega_{1}$ she also considers possible $\omega_{2}$, which is not included in $R$. Formally: $P_{A}\left(\omega_{1}\right)=\left\{\omega_{1}, \omega_{2}\right\} \not \subset R$. In $\omega_{1}$, however, Ann does believe $S$, because in every state she regards as possible in $\omega_{2}-$ that is, in $P_{A}\left(\omega_{1}\right)=\left\{\omega_{1}, \omega_{2}\right\}$-the event $S$ occurs: $P_{A}\left(\omega_{1}\right)=\left\{\omega_{1}, \omega_{2}\right\} \subseteq S$.

As it happens, beliefs may turn out to be incorrect, and in this case the event believed by the individual does not occur. The state-space model is flexible enough to capture this situation. Imagine that the possibility correspondence of Ann in $\omega_{4}$ is as follows: $P_{A}\left(\omega_{4}\right)=\left\{\omega_{1}\right\}$. This means that if $v=4$, for some reason Ann erroneously believes that $v=1$. Consider again the event $S$ " $v \leq 2$ ". In $\omega_{4}$, does Ann believe $S$ ? Yes, she does, because in $\omega_{1}$, the only state she erroneously regards as possible in $\omega_{4}, v$ is not greater than 2. Formally: $P_{A}\left(\omega_{4}\right)=\left\{\omega_{1}\right\} \subseteq S$. However, this belief is false.

As mentioned in the Introduction, the primary characteristic that distinguishes knowledge from belief is that while belief can be false, knowledge is always true. In the state-space model this property of knowledge is captured by the inclusion of the true state of the world $\omega$ among those the individual regards as possible in $\omega$ itself.

Formally, knowledge requires that $\omega \in P(\omega)$. In fact, if $\omega \in P(\omega)$, when at $\omega$ the individual believes event $E$, i.e., when $P(\omega) \subseteq E$, the event $E$ actually occurs in $\omega$, i.e., $\omega \in E$. Based on this, we can define a knowledge operator $K: 2^{\Omega} \rightarrow 2^{\Omega}$, which is a refinement of the belief operator $B$, as follows: $K(E)=\{\omega \in \Omega: \omega \in P(\omega) \wedge P(\omega) \subseteq E\}$.

In our example, when $v=1$ Ann not only believes that the event $v$ is not greater than 2, but her belief is true, and so she knows that event. On the contrary, when $v=4$ Ann believes that $v$ is not greater than 2, but since $\omega \notin P(\omega)$ she is wrong.

In the literature on the state-space model, the terminology concerning belief and knowledge is ambiguous. Sometimes the term "knowledge" is used in a broad sense to express what in this paper is called "belief". In this broad sense, knowledge may be false. This usage, however, seems at odds with the basic philosophical notion of 
knowledge according to which truthfulness is a necessary condition for knowledge. At other times the term "belief " is used in a strict sense to express what in this paper is called "knowledge", that is, it is assumed that beliefs are always true. This assumption is called the truth axiom, and formally it requires that $\omega \in P(\omega)$ for every state of the world $\omega$ belonging to $\Omega$. This second approach also seems problematic, because eliminating false beliefs may prevent us from understanding the numerous situations in which actual individuals have indeed false beliefs about economic variables. I therefore maintain the distinction between beliefs, which can be false, and knowledge, which is truthful by definition.

It is important to stress that for the argument made in the paper the truth axiom is not needed. The intension-extension issues addressed here concern both true and false beliefs, i.e., the operator $B$ as well as the operator $K$. In effect, we could have limited our discussion to the general case of belief, without dealing with the particular case of true belief or knowledge. But since the literature on the state-space model generally refers to knowledge, it seemed appropriate to discuss knowledge explicitly. ${ }^{9}$

\section{BELIEF AND KNOWLEDGE, WITHOUT INTENSION}

In the Introduction and Section 1 we have discussed intension and extension as related to linguistic expressions. In the state-space model we do not find linguistic expressions but subsets of $\Omega$ called events, which are however interpreted as set-theoretic images of linguistic expressions like "it rains" or " $v$ is not greater than 2 ". The formal link between a given linguistic expression and its set-theoretic image is provided by a correspondence that associates the expression at issue with the subset of states of the world where the expression is true. In this set-theoretic translation, linguistic expressions come to be identified with their extensional correlates in $\Omega$, and thus lose their intensional dimension.

For instance, in our illustration of the state-space model we have labeled the event " $v$ is not greater than 2" as $S$, and observed that $S$ occurs at states $\omega_{1}$ and $\omega_{2}$. Consider now the event " $v$ is not greater

\footnotetext{
${ }^{9}$ In addition to the truth axiom, the state-space literature generally imposes a second axiom that warrants some nice properties of the knowledge operator $K$, and formally states that if $\omega^{\prime} \in P(\omega)$, then $P\left(\omega^{\prime}\right)=P(\omega)$. Our discussion is also independent of this second axiom.
} 
than the 12th root of 4096", which can be labeled as T. T exhibits an intension distinct from $S$, but in fact the two events have the same extension-for the 12th root of 4096 is 2-and thus the same set-theoretic image, given by $\left\{\omega_{1}, \omega_{2}\right\}$. By the axiom of extensionality of the Zermelo-Fraenkel set theory, $S$ and $T$ are equal: $S=T$.

Consider now the set-theoretic operators $B$ and $K$, which map subsets of $\Omega$ into other subsets of $\Omega$. By their very nature, $B$ and $K$ map and cannot avoid mapping equal input-sets into equal output-sets:

If $S=T$, then $B(S)=B(T)$ and $K(S)=K(T)$.

Therefore, since in the state-space model the events " $v \leq 2$ " and " $v \leq \sqrt[12]{4096}$ " have the same extensional, set-theoretic correlate, if Ann believes (knows) one event, she also believes (knows), and cannot avoid believing (knowing), the other event. ${ }^{10}$

This implication illustrates, albeit in the over-simplified setting of our numerical example, the failure of the operators $B$ and $K$ to account for framing effects. The events $v \leq 2$ and $v \leq \sqrt[12]{4096}$ are extensionally equal but intensionally different, and we may well imagine that Ann reasonably believes that $v \leq 2$ without believing that $v \leq \sqrt[12]{4096}$. But, because of their extensional character, the operators $B$ and $K$ automatically implement the substitutivity principle, and therefore are unable to accounting for the intensional difference between $v \leq 2$ and $v \leq \sqrt[12]{4096}$.

In attempting to connect this abstract numerical example with more realistic economic situations, we may interpret $v$ as any parameter relevant to the individual, while " $v \leq 2$ " and " $v \leq \sqrt[12]{4096}$ " could stand for, respectively, a "plain" way and a "convoluted" way of presenting that parameter. For instance, $v$ could be associated with the probability of defaulting on a loan, while $v \leq 2$ and $v \leq \sqrt[12]{4096}$ could stand, respectively, for a "plain" and a "convolute " way in which a bank can present that likelihood to a potential subscriber of the loan. We may imagine that the individual's beliefs about $v$, and accordingly

\footnotetext{
${ }^{10}$ Monderer and Samet (1989) and others added a probability structure to the statespace model in order to express different degrees of belief about an event, that is, to express statements such as "Ann believes event $E$ with probability at least $p$ ". Since probabilities apply to sets, the problematic feature of the basic state-space discussed here concerns also its probabilistic extensions: if Ann believes that $v \leq 2$ with probability at least $p$, she also believes that $v \leq \sqrt[12]{4096}$ with probability at least $p$.
} 
her willingness to subscribe the loan, depend on the way the bank presents to her the likelihood of defaulting. The point I want to make here is that the operator $B$ fails to capture the intensional difference between the plain and convoluted framings of that likelihood.

\section{INTENSION AND EXTENSION FOR INTERACTIVE BELIEFS AND KNOWLEDGE}

Interactive beliefs and interactive knowledge are, respectively, beliefs or knowledge that an individual has about what other individuals believe or know about the world. Interactive beliefs and knowledge are often economically relevant since in many cases individuals take action on the basis of them. Consider, for example, a standard principal-agent situation in which both the principal and the agent are interested in a certain event $E$ that typically is the result of the agent's effort. If the agent believes that the principal does not know his effort he may choose a low effort, while if the agent believes that the principal knows his effort he will probably choose a high effort.

In effect, there are multiple levels of interactive beliefs and knowledge. The first level is the one discussed above: it concerns what an individual believes or knows about what other individuals believe or know about the world. The second level concerns what an individual believes or knows about what other agents believe or know about her/his beliefs or knowledge of the world. The staircase of levels of interactive beliefs and knowledge escalates in a predictable way, and game theory has shown that interactive beliefs and knowledge of higher or even infinite levels are of great consequence in strategic environments (see, e.g., Rubinstein 1989).

The state-space model makes it possible, not only to represent what individuals believe or know about the world, but also to represent their interactive beliefs and knowledge at any possible level. However, the model's difficulties in capturing the intensional difference between extensionally equal events and the puzzling consequences this generates extend also to interactive beliefs and knowledge. To keep things simple, we focus here on the first level of interactive beliefs and knowledge.

Let us add another agent-Bob-to our illustration of the model. Imagine that Bob is interested in the same variable $v$ (taking values from 1 to 4) in which Ann is interested. Assume that Bob's possibility correspondence $P_{B}$ is as follows: 


$$
P_{B}\left(\omega_{1}\right)=P_{B}\left(\omega_{2}\right)=\left\{\omega_{1}, \omega_{2}\right\}, P_{B}\left(\omega_{3}\right)=P_{B}\left(\omega_{4}\right)=\left\{\omega_{3}, \omega_{4}\right\} .
$$

Consider now the event $U$ " $v \leq 3$ " that occurs at states $\omega_{1}, \omega_{2}$, and $\omega_{3}: U=\left\{\omega_{1}, \omega_{2}, \omega_{3}\right\}$. In which states of the world does Bob believe that $v \leq 3$ ? It easy to show that these states are $\omega_{1}$ and $\omega_{2}$. That "Bob believes that $v \leq 3$ " is itself an event occurring in those two states, expressed as $B_{B}(U): B_{B}(U)=\left\{\omega_{1}, \omega_{2}\right\}$. Notice that the event $S$ " $v \leq 2$ " that we dealt with in the previous sections, and the event $B_{B}(U)$ "Bob believes that $v \leq 3$ ", occur in exactly the same states of the world $\omega_{1}$ and $\omega_{2}: B_{B}(U)=S$. Therefore, although the two events are intensionally distinct, their set-theoretic image in our simple Ann-Bob state-space is the same.

Imagine now that the true state of the world is $\omega_{1}$. We have just seen that at $\omega_{1}$ Bob does believe that $v \leq 3$. At this point, interactive beliefs enters the scene and we can ask: at $\omega_{1}$, does Ann believe that Bob believes that $v \leq 3$ ?

From an intuitive viewpoint, the answer is that it depends on what Ann believes at $\omega_{1}$ about the way information is imparted to Bob. Since in the state-space model the way information is imparted to an agent is formally represented by his possibility correspondence $P$, one may be tempted to formulate the same answer by saying that whether at $\omega_{1}$ Ann believes that Bob believes that $v \leq 3$ depends on whether at $\omega_{1}$ Ann knows Bob's possibility correspondence $P_{B}$. However, this formulation is misleading because it mixes up the viewpoint of the modeler with that of the agents in the model. As observed in Section 2, possibility correspondences are in fact just a tool that the modeler employs to encode and represent the agents' beliefs and knowledge, not something that the agents need to be aware of or even know. Therefore, possibility correspondences exist for the model-maker but need not exist for the agents in the model, and thus reasoning as if he agents may or may not know them is confusing.

At any rate, let us return to the intuitive answer to the question: "At $\omega_{1}$, does Ann believe that Bob believes that $v \leq 3$ ?". In Section 2 we saw that at $\omega_{1}$ Ann believes that the true state is $\omega_{1}$ or $\omega_{2}$. If Ann believes that in $\omega_{1}$ and $\omega_{2}$ Bob regards as possible both $v=1$ and $v=2$ (as is, in fact, the case for Bob), then in $\omega_{1}$ Ann can conclude that Bob believes that $v \leq 3$. By contrast, if Ann has no clue about the way 
information is imparted to Bob in $\omega_{1}$ and $\omega_{2}$ then she has no idea about what Bob believes in these two states and so cannot conclude that Bob believes that $v \leq 3$. In other words, the intuitive answer to the above question turns out to be that we need to make some additional assumption about Ann's information about Bob's information in order to answer the question about Ann's beliefs about Bob's beliefs.

The formal answer offered by the state-space model is however different: since in $\omega_{1}$ Ann believes $S$, i.e., $\omega_{1} \in B_{A}(S)$, and the set of states where Bob believes $U$ coincides with $S$, i.e., $S=B_{B}(U)$, then by the substitutivity principle $\omega_{1} \in B_{A}\left(B_{B}(U)\right)$. In the state-space model the latter expressions means that in $\omega_{1}$ Ann believes that Bob believes that $v \leq 3$. And this conclusion is independent of any additional assumption as to Ann's information about the way information is imparted to Bob. One may add that in our Ann-Bob example, in $\omega_{1}$ and $\omega_{2}$ both Ann's and Bob's beliefs are true, so that in $\omega_{1}$ Ann not only believes but also knows that Bob knows that $v \leq 3$.

This puzzling result is again due to the fact that the operators $B$ and $K$ are unable to distinguish between intension and extension. In fact, although intensionally distinct, in our model the events " $v \leq 2$ " and "Bob believes that $v \leq 3$ " are extensionally equal. Therefore $B$ and $K$ automatically implement the substitutivity principle even if, as in this case, it is unwarranted.

Aumann has always claimed that, if the state-space $\Omega$ is defined in an appropriate way, each agent knows by construction how the information is imparted to other agents, and that this knowledge "is not an assumption, but a 'theorem', a tautology; it is implicit in the model itself" (Aumann 1987, 9). ${ }^{11}$ Here I argue that the fundamental reason for this counterintuitive feature of the state-space formalism when applied to interactive beliefs and knowledge lies in its extensional nature, which prevents the model from telling apart events that are extensionally equal but intensionally distinct.

This feature of $B$ and $K$ may preclude the state-space model from capturing the uncertainty that an agent has about the way the information is imparted to other agents, although that uncertainty is a

\footnotetext{
${ }^{11}$ For a discussion of Aumann's argument and its possible limitations, see Aumann 1999; Heifetz and Samet 1998; Heifetz 1999; Fagin, et al. 1999; Aumann and Heifetz 2002; Cubitt and Sugden 2003.
} 
key element of the situation at issue and crucially affects the agents' behavior.

For instance, we can re-interpret our Ann-Bob model as representing a duopoly game, whereby the two agents are the duopolists, $v$ is the level of next period's market demand, and lower levels of $v$ correspond to lower expected demand levels. At $\omega_{1}$, Ann receives a signal that in the next period the market demand will be quite low $(v=1$ or $v=2)$ and has to decide how much to produce. If Ann considers it possible that Bob erroneously believes that the demand will be very high $(v=4)$, she is afraid that Bob could produce a large output, and therefore she might cautiously choose an extremely low output level. This behavior and its motivations cannot be captured by our state-space model because, as shown above, in that model Ann knows and cannot avoid knowing that Bob knows that $v \leq 3$, and thus that he rules out a very high demand.

One may easily imagine many other economically relevant situations in which the failure to capture the agents' uncertainty about the information that other agents may have through the operators $B$ and $K$ renders the use of the state-space model problematic.

\section{Possible SOlUTIONS}

Besides its failure to capture the intensional dimension of linguistic expressions, the state-space model displays other implausible features: it implies the monotonicity of beliefs and knowledge and precludes agents' unawareness. Since some solutions to these latter limitations have been proposed, one may wonder whether there are ways to modify the operators $B$ and $K$ and/or the state-space model in such a way that they become capable of capturing the difference between events that are extensionally equivalent but intensionally distinct. Here I discuss three possible ways in which one may imagine that this could be done.

First, the state-space model can make room for unawareness and block monotonicity by introducing a further set-theoretic operator $A: \Omega \rightarrow 2^{2^{\Omega}}$, that associates each state of world $\omega$ with a collection of subsets of $\Omega$. A can be interpreted as an awareness correspondence that associates each state $\omega$ with the events that the agent is able to 
figure out in $\omega .^{12}$ Based on the awareness operator $A$, one may define a modified belief operator $B^{\prime}$ as follows:

$$
B^{\prime}(E)=\{\omega \in \Omega: P(\omega) \subseteq E \wedge E \in A(\omega)\} .
$$

The interpretation of $B^{\prime}$ is that believing an event requires not only that the event occurs in every state the agent regards as possible, i.e., $P(\omega) \subseteq E$, but also that the agent can figure out the event, i.e. $E \in A(\omega)$. For instance, if at $\omega_{1}$ Ann is not able to figure out the meaning of "12th root of 4096", then although in all states she regards as possible at $\omega_{1}$ the event $T$ " $v \leq \sqrt[12]{4096}$ " occurs, she does not believe it.

The operator $A$ can also be used to block monotonicity. Monotonicity states that if event $E$ implies event $F-$ a situation that in set-theoretical terms is expressed by $E \subseteq F$-then believing event $E$ implies believing its implications $F$, i.e., $B(E) \subseteq B(F) .{ }^{13}$ Operator $A$ can block monotonicity as follows: if $E \subseteq F$ and $E \in A(\omega)$, but $F \notin A(\omega)$, then $B^{\prime}(E)$ is not a subset of $B^{\prime}(F)$. In words, if event $E$ implies event $F$ and the agent is aware of $E$ but unaware of $F$, then her believing $E$ does not imply that she also believes the implications of $E$.

Is it also possible to use the operator $A$ to block the automatic implementation of the substitutivity principle by the operators $B$ and $K$, and thus account for the possible intensional difference of extensionally equivalent events? The answer appears to be in the negative, because $A$ too is extensional in nature and therefore cannot distinguish between extensionally equivalent sets: if $E=F, E \in A(\omega)$ if and only if $F \in A(\omega)$.

In our example, the extensional nature of operator $A$ has the problematic implication that Ann is able to figure out the event $S$ " $v \leq 2$ " if and only if she is able to figure out the extensionally equivalent event $T$ " $v \leq \sqrt[12]{4096}$ ". However, one may well imagine that Ann is aware of " $v \leq 2$ " while ignoring " $v \leq \sqrt[12]{4096}$ ". Since the operator $A$ cannot distinguish between extensionally equivalent sets, not even

\footnotetext{
${ }^{12}$ The definition of the awareness operator proposed here follows Fagin, et al. 1995; for other ways of modeling unawareness, see Modica and Rustichini 1999; Halpern 2001; Heifetz, et al. 2006; Li, 2009.

${ }^{13}$ The property of operators $B$ and $K$ discussed in this paper, namely that if $E=F$ then $B(E)=B(F)$ and $K(E)=K(F)$, is strictly weaker than monotonicity. The latter property reduces to the former in the particular case when $E=F$.
} 
the modified belief operator $B^{\prime}$ based on $A$ can distinguish between them: if $E=F$, then $B^{\prime}(E)=B^{\prime}(F)$. The same holds for the modified knowledge operator $K^{\prime}$ that can be built upon operator $B^{\prime}$ in the predictable way.

The discussion concerning the operators $A, B^{\prime}$, and $K^{\prime}$ suggests that attempts to capture the intensional difference between extensionally equivalent events by introducing into the state-space model some new set-theoretic operator like $A$ are bound to fail. In fact, any operator of this kind would map extensionally equal inputs into the same output, and so would be unable to overcome the problem.

A second possible way out of the problem consists of re-defining the set of the states of the world $\Omega$, not as the external and omniscient model-maker sees it, but as each agent subjectively views $\Omega$. From this point of view, the problem does not lie in the set-theoretic nature of the operators $B$ and $K$, but in a specification of $\Omega$ that is not sufficiently fine-grained to capture all the relevant aspects of the situation.

For instance, if for Ann $v=2$ and $v \leq \sqrt[12]{4096}$ are two distinct events, then her subjective state-space $\Omega_{A}$ is finer-grained than the modelmaker's state-space $\Omega$ and includes, besides the state $\omega_{2}$ where $v=2$, also a state $\omega_{5}$ where $v \leq \sqrt[12]{4096}: \Omega_{A}=\left\{\omega_{1}, \omega_{2}, \omega_{3}, \omega_{4}, \omega_{5}\right\}$. At $\omega_{2}$ and $\omega_{5}$, Ann may regard as possible different states of the world. For instance, it could be that $P_{A}\left(\omega_{2}\right)=\left\{\omega_{1}, \omega_{2}\right\}$ and $P_{A}\left(\omega_{5}\right)=\left\{\omega_{5}\right\}$, so that at $\omega_{2}$ Ann believes that $v \leq 2$ without believing that $v \leq \sqrt[12]{4096}$.

I see two problems in the modeling strategy based on the subjective re-definition of $\Omega$. First, defining the subjective state-space of each agent in the model requires that the modeler knows how each agent sees the world and the intensional meaning the agent gives to different expressions. This appears more demanding than defining the state-space as it is "objectively", or at least, as it is from the modeler's view. For instance, in our over-simplified numerical example, besides $v \leq \sqrt[12]{4096}$ there are countless other alternative intensions for $v=2$, such as $v=5-3 ; \quad v=\left(5^{2}-3^{2}\right) / 2^{3} ; \quad v=\sqrt[13]{8192}, \quad v=2 \cos (2 \pi)$, $v=5 ! /\left(3^{3}+33\right)$. Which of these expressions are understood by Ann as equivalent to $v=2$, and thus could be identified with the state $\omega_{2}$ ? And which ones, instead, require the addition of further states of the world to Ann's subjective state-space $\Omega_{A}$ ? 
Second, fine-grained subjective state-spaces quickly become very complex and thus could easily make the state-space model difficult to handle for the purposes of economic analysis. For instance, we saw that when $v=1$ Ann knows that $v \leq 2$. Imagine now that, when $v=1$, Ann has little clue about Bob's information and regards as possible four situations: i) Bob knows exactly whether $v=1$ or $v=2$; ii) like herself, Bob only knows that $v \leq 2$; iii) Bob is less informed than herself and can only rule out that $v=4$; iv) Bob erroneously believes that $v>2$.

To model this situation we can split the state $\omega_{1}$ into four states $\omega_{1}^{i}, \omega_{1}^{i i}, \omega_{1}^{i i i}, \omega_{1}^{i v}$, each of which specifies the value of $v$ as well as Bob's information about the value of $v$. Thus, in $\omega_{1}^{i}, v=1$ and Bob knows whether $v=1$ or $v=2$; in $\omega_{1}^{i i}, v=1$ and Bob knows that $v \leq 2$; in $\omega_{1}^{i i i}, v=1$ and Bob knows that $v \neq 4$; in $\omega_{1}^{i v}, v=1$ and Bob believes that $v>2$. If Ann is uncertain about Bob's information, she is unable to distinguish between $\omega_{1}^{i}, \omega_{1}^{i i}, \omega_{1}^{i i i}$, and $\omega_{1}^{i v}$, and whichever state is the true one she regards all four of them as possible:

$$
P_{A}\left(\omega_{1}^{i}\right)=P_{A}\left(\omega_{1}^{i i}\right)=P_{A}\left(\omega_{1}^{i i i}\right)=P_{A}\left(\omega_{1}^{i \nu}\right)=\left\{\omega_{1}^{i}, \omega_{1}^{i i}, \omega_{1}^{i i i}, \omega_{1}^{i \nu}\right\} .
$$

By modeling Ann's beliefs and uncertainty this way, we can avoid the puzzling result that when $v=1$, Ann not only knows that $v \leq 2$ but also that Bob knows that $v \leq 3$. In fact, when $v=1$ Ann cannot rule out $\omega_{1}^{i v}$, at which state, however, Bob believes that $v>2$.

The cost of this modeling strategy is that we have to add three states of the world to the "objective" state-space just to account for Ann's uncertainty about Bob's information about the value of $v$, in the particular case when $v=1$. If we also want to account for Ann's possible uncertainty about Bob's information about the value of $v$ when $v=2$, $v=3$, or $v=4$, we would have to add further states to Ann's statespace $\Omega_{A}$.

We may also want to consider the first level of interactive beliefs and uncertainty, and model Ann's uncertainty about what Bob believes about her information about the value of $v$. This would require adding many more elements to $\Omega_{A}$. Modeling higher levels of interactive beliefs and uncertainty would require adding even more states to $\Omega_{A}$. And, if all this were not enough, we might also want to model Bob's subjective state-space $\Omega_{B}$, his uncertainty about Ann's information about the value of $v$, his uncertainty about what Ann believes about his 
information about the value of $v$, and so forth. In brief, properly fine-grained subjective state-spaces easily become intractable objects that as such may be of little use in economic theorizing. ${ }^{14}$

A third possible way to capture the intensional difference between extensionally equivalent events is to abandon the state-space and the set-theoretic modeling of beliefs and knowledge, and adopt the so-called logic-based or syntactic approach to beliefs and knowledge that is prevalent among logicians and philosophers. The building blocks of this approach are formulas, which correspond to events and are expressed by alphabetical letters, and epistemic operators such as $b$ (for belief) or $k$ (for knowledge) that associate formulas with other formulas according to certain axioms that can be stated in an explicit way. ${ }^{15}$

The fundamental difference between the operators $B$ and $K$ of the state-space model and the operators $b$ and $k$ of the syntactic approach is that, in principle, the latter can be axiomatized so as to avoid the automatic implementation of the substitutivity principle. More specifically, one can design a syntactic system in which, although two formulas $e$ and $f$ coincide, even in every imaginable universe, this does not imply that when individual $i$ believes or knows one of them she must also believe or know the other.

Formally, although $e \leftrightarrow f$ and $b_{i} e$, it can be the case that $\neg b_{i} f$ ( $\leftrightarrow$ and $\neg$ are, respectively, the logical symbols for "necessary and sufficient condition", and "negation"). However, de facto, even those economists who have employed the syntactic approach have maintained the substitutivity principle, that is, they have still posited that if $e \leftrightarrow f$ and $b_{i} e$, then $b_{i} f$ (see, e.g., Lismont and Mongin 1994a, 1994b, 1995, 2003; Ferrante 1996; Dardi 2004; Heifetz, et al. 2008).

In part, the maintenance of the substitutivity principle is due to the fact that economists have adopted the syntactic approach in order to overcome other implausible features of the state-space model, specifically, that it implies the monotonicity of beliefs and knowledge or that it precludes unawareness, rather than its treating as equal expressions that are intensionally distinct. But another reason why economists have maintained the substitutivity principle is that not even

\footnotetext{
${ }^{14}$ Actually there are some situations in which no number of splits of the states of the world is sufficiently large to exhaust all interactive uncertainty of the agents, that is, to properly fine-grain the model. See Hart, et al. 1996; Heifetz and Samet 1998; Heifetz 1999; Fagin, et al. 1999; Aumann and Heifetz 2002.

${ }^{15}$ For an introduction to the syntactic approach, see Fagin, et. al. 1995; Aumann 1999.
} 
logicians and philosophers agree as to how it might be given up. As mentioned in Section 1, while various systems of intensional logic aimed at capturing explicitly the intension-extension difference have been put forward in the last sixty-five years, none of them has ever gained general acceptance.

From a sociological viewpoint, we may add that the syntactic approach requires a familiarity with the language, modeling techniques, and key results of epistemic logic, none of which form part of the typical background of economists. The significant fixed costs associated with acquiring that familiarity discourage economists from using the syntactic approach.

If we put together the difficulty of giving up the substitutivity principle, even in the syntactic approach, with economists' lack of familiarity with epistemic logic, it appears less clear that adopting the syntactic approach is the best move to overcome the difficulties of the state-space model in accounting for extensions with different intensions.

To sum up the discussion: attempts to capture the intensional difference between extensionally equivalent events by introducing into the state-space model some new set-theoretic operator like $A$ appear bound to fail; the other two routes-a subjective re-definition of $\Omega$ and the move to the syntactic approach-are more promising, but each of them contains significant drawbacks.

\section{CoNCLUSIONS}

I have attempted to draw the attention of economists to a cluster of problems that have proved important to philosophers, at least since the end of the nineteenth century, and are related to the relationship between the intension and the extension of a linguistic expression. In particular, philosophers have long understood that such a relationship becomes opaque in a number of contexts, particularly those involving belief and knowledge. In the paper I have shown that the problems related to the intension and extension of an expression are relevant also for economics, and particularly for the model of belief and knowledge prevailing in this discipline, namely the state-space model introduced by Aumann in 1976.

In particular, I have argued that, because of its set-theoretic nature, the state-space model tends to miss the difference between expressions that have the same extension but distinct intensions. This feature of the 
model generates a number of puzzling results that concern not only what individuals believe or know about the world, but also interactive beliefs and knowledge. I have also attempted to connect these puzzling results with two issues that are relevant for economic theory even beyond the state-space model, namely framing effects and the distinction between model-maker's and agents' viewpoints in the model.

The limitations of the state-space model do not imply that this model should be abandoned. Indeed, I have argued that the two alternatives that appear practicable present significant drawbacks: a properly fine-grained subjective state-space $\Omega$ easily becomes intractable, while dismissing the substitutivity principle is de facto tricky even in the syntactic approach. At the present moment, I cannot envisage any general method to assess the trade-off between the advantages and drawbacks of these alternatives.

\section{REFERENCES}

Arrow, Kenneth J. 1982. Risk perception in psychology and economics. Economic Inquiry, 20 (1): 1-9.

Aumann, Robert J. 1976. Agreeing to disagree. Annals of Statistics, 4 (6): 1236-1239.

Aumann, Robert J. 1987. Correlated equilibrium as an expression of Bayesian rationality. Econometrica, 55 (1): 1-18.

Aumann, Robert J. 1999. Interactive epistemology I: knowledge. International Journal of Game Theory, 28 (3): 263-300.

Aumann, Robert J., and Aviad Heifetz. 2002. Incomplete information. In Handbook of game theory, Vol. III, eds. Robert J. Aumann, and Sergiu Hart. Amsterdam: North Holland, 1665-1686.

Bacharach, Michael O. 1986. The problem of agent's beliefs in economic theory. In Foundations of economics: structures of inquiry and economic theory, eds. Mauro Baranzini, and Roberto Scazzieri. New York: Blackwell, 175-203.

Battigalli, Pierpaolo, and Giacomo Bonanno. 1999. Recent results on belief, knowledge and the epistemic foundations of game theory. Research in Economics, 53 (2): 149225.

Bealer, George. 1998. Intensional entities. In Routledge Encyclopedia of Philosophy, Vol. 4, ed. Edward Craig. London: Routledge, 803-807.

Bourgeois-Gironde, Sacha, and Raphaël Giraud. 2009. Framing effects as violations of extensionality. Theory and Decision, 67 (4): 385-404.

Brandenburger, Adam. 1992. Knowledge and equilibrium in games. Journal of Economic Perspectives, 6 (4): 83-101.

Cantor, Georg. 1883. Grundlagen einer allgemeinen Mannigfaltigkeitslehre. Leipzig: Teubner.

Carnap, Rudolf. 1947. Meaning and necessity. Chicago: University of Chicago Press.

Church, Alonzo. 1951. A formulation of the logic of sense and denotation. In Structure, method and meaning, eds. Paul Henle, Horace M. Kallen, and Susanne K. Langer. New York: The Liberal Arts Press, 3-24. 
Church, Alonzo. 1973. Outline of a revised formulation of the logic of sense and denotation (Part I). Noûs, 7 (1): 24-33.

Church, Alonzo. 1974. Outline of a revised formulation of the logic of sense and denotation (Part II). Noûs, 8 (2) 135-156.

Cubitt, Robin P., and Robert Sugden. 2003. Common knowledge, salience and convention: a reconstruction of David Lewis' game theory. Economics and Philosophy, 19 (2): 175-210.

Dardi, Marco. 2004. Modello economico e modello dell'agente. In Economia senza gabbie, eds. Nicolò Bellanca, Marco Dardi, and Tiziano Raffaelli. Bologna: il Mulino, 489-520.

Dedekind, Richard. 1888. Was sind und was sollen die Zahlen?. Braunschweig: Vieweg.

Dekel, Eddie, Barton L. Lipman, and Aldo Rustichini. 1998. Standard state-space models preclude unawareness. Econometrica, 66 (1): 159-73.

Dekel, Eddie, and Faruk Gul. 1997. Rationality and knowledge in game theory. In Advances in economics and econometrics: theory and applications. Seven World Congress, Vol. 1, eds. David M. Kreps, and Kenneth F. Wallis. Cambridge: Cambridge University Press, 87-172.

Fagin, Ronald, John Geanakoplos, Joseph Y. Halpern, and Moshe Y. Vardi. 1999. The hierarchical approach to modeling knowledge and common knowledge. International Journal of Game Theory, 28 (3): 331-365.

Fagin, Ronald, Joseph Y. Halpern, Yoram Moses, and Moshe Y. Vardi. 1995. Reasoning about knowledge. Cambridge (MA): MIT Press.

Ferrante, Vittorioemanuele. 1996. A sound interpretation of minimality properties of common belief in minimal semantics. Theory and Decision, 41 (2): 179-185.

Ferreirós, José. 2007. Labyrinth of thought. Berlin: Birkhäuser.

Fitting, Melvin. 2004. First-order intensional logic. Annals of Pure and Applied Logic, 127 (1-3): 171-193.

Fitting, Melvin. 2011. Intensional logic. In Stanford Encyclopedia of Philosophy (Spring 2011 Edition), ed. Edward N. Zalta.

http://plato.stanford.edu/archives/spr2011/entries/logic-intensional/ (accessed July 6, 2012).

Fraenkel Abraham. 1923. Einleitung in die Mengenlehre. Berlin: Springer.

Frege, Gottlob. 1948 [1892]. Sense and reference. Philosophical Review, 57 (3): 209-230.

Gallin, Daniel. 1975. Intensional and higher-order modal logic. Amsterdam: NorthHolland.

Garson, James W. 1998. Intensional logics. In Routledge Encyclopedia of Philosophy, Vol. 4, ed. Edward Craig. London: Routledge, 807-811.

Gettier, Edmund L. 1963. Is justified true belief knowledge? Analysis, 23 (6): 121-123.

Giocoli, Nicola. 2001. Fixing the point: the contribution of early game theory to the tool-box of modern economics. Journal of Economic Methodology, 10 (1): 1-39.

Halpern, Joseph. 2001. Alternative semantics for unawareness. Games and Economic Behavior, 37 (2): 321-339

Hart, Sergiu, Aviad Heifetz, and Dov Samet. 1996. "Knowing whether", "Knowing that", and the cardinality of state spaces. Journal of Economic Theory, 70 (1): 249-256.

Heifetz, Aviad. 1999. How canonical is the canonical model? A comment on Aumann's interactive epistemology. International Journal of Game Theory, 28 (3): 435-442.

Heifetz, Aviad, and Dov Samet. 1998. Knowledge spaces with arbitrarily high rank. Games and Economic Behavior, 22 (2): 260-273. 
Heifetz, Aviad, Martin Meier, and Burkhard C. Schipper. 2006. Interactive unawareness. Journal of Economic Theory, 130 (1): 78-94.

Heifetz, Aviad, Martin Meier, and Burkhard C. Schipper. 2008. A canonical model for interactive unawareness. Games and Economic Behavior, 62 (1): 304-324.

Kahneman, Daniel, and Amos Tversky. 1984. Choices, values, and frames. American Psychologist, 39 (4): 341-350.

Li, Jing. 2009. Information structures with unawareness. Journal of Economic Theory, 144 (3): 977-993.

Lismont, Luc, and Philippe Mongin. 1994a. On the logic of common belief and common knowledge. Theory and Decision, 37 (1): 75-106.

Lismont, Luc, and Philippe Mongin. 1994b. A non-minimal but very weak axiomatization of common belief. Artificial Intelligence, 70 (1-2): 363-374.

Lismont, Luc, and Philippe Mongin. 1995. Belief closure: a semantics of common knowledge for modal propositional logic. Mathematical Social Sciences, 30 (2): 127 153.

Lismont, Luc, and Philippe Mongin. 2003. Strong completeness theorems for weak logics of common belief. Journal of Philosophical Logic, 32 (2): 115-137.

McKay, Thomas, and Michael Nelson. 2010. Propositional attitude reports. In Stanford Encyclopedia of Philosophy (Winter 2010 Edition), ed. Edward N. Zalta.

http://plato.stanford.edu/archives/win2010/entries/prop-attitude-reports/ (accessed July 10, 2012).

Modica, Salvatore, and Aldo Rustichini. 1999. Unawareness and partitional information structures. Games and Economic Behavior, 27 (2): 265-298.

Monderer, Dov, and Dov Samet. 1989. Approximating common knowledge with common belief. Games and Economic Behavior, 1 (2): 170-190.

Montague, Richard. 1960. On the nature of certain philosophical entities. The Monist, 53 (2): 159-194.

Montague, Richard. 1970. Pragmatics and intensional logic. Synthese, 22 (1-2): 68-94.

Neumann, John von. 1928. Die Axiomatisierung der Mengenlehre. Mathematische Zeitschrift, 27: 669-752.

Osborne, Martin J., and Ariel Rubinstein. 1994. A course in game theory. Cambridge (MA): MIT Press.

Rubinstein, Ariel. 1989. The electronic mail game: strategic behavior under "almost common knowledge". American Economic Review, 79 (3): 385-391.

Samuelson, Larry. 2004. Modeling knowledge in economic analysis. Journal of Economic Literature, 42 (2): 367-403.

Steup, Matthias. 2012. The analysis of knowledge. In Stanford Encyclopedia of Philosophy (Summer 2012 Edition), ed. Edward N. Zalta.

http://plato.stanford.edu/archives/sum2012/entries/knowledge-analysis/ (accessed July 9, 2012).

Tversky, Amos, and Daniel Kahneman. 1981. The framing of decisions and the psychology of choice. Science, 211 (4481): 453-458

Tversky, Amos, and Daniel Kahneman. 1986. Rational choice and the framing of decisions. Journal of Business, 50 (4): S251-S278

Vilks, Arnis. 1995. On mathematics and mathematical economics. Greek Economic Review, 17 (2): 177-204. 
Wakker, Peter P. 1999. Justifying Bayesianism by dynamic decision principles. Working paper. Leiden University, The Netherlands.

Weintraub, E. Roy. 2002. How economics became a mathematical science. Durham (NC): Duke University Press.

Zalta, Edward N. 1988. Intensional logic and the methaphysics of intensionality. Cambridge (MA): MIT Press.

Zermelo, Ernst. 1908. Untersuchungen über die Grundlagen der Mengenlehre. Mathematische Annalen, 65 (2): 261-281.

Ivan Moscati is associate professor of economics at the University of Insubria, Varese, and teaches history of economic thought at Bocconi University, Milan. His research focuses on the history and methodology of economics. He is currently working on a research project on the history of utility measurement. The first installment of the project is forthcoming in History of Political Economy under the title "Were Jevons, Menger, and Walras really cardinalists?: on the notion of measurement in utility theory, psychology, mathematics and other disciplines, ca. 1870-1910".

Contact e-mail: <ivan.moscati@uninsubria.it> 